Algebraic 83 Geometric $\mathcal{T}$ opology

Volume 5 (2005) 1315-1324

Published: 6 October 2005

ATG

\title{
Twisted Alexander polynomials and surjectivity of a group homomorphism
}

\author{
Teruaki Kitano \\ MASAAKI SUZUKI \\ MASAAKI WADA
}

\begin{abstract}
If $\varphi: G \rightarrow G^{\prime}$ is a surjective homomorphism, we prove that the twisted Alexander polynomial of $G$ is divisible by the twisted Alexander polynomial of $G^{\prime}$. As an application, we show non-existence of surjective homomorphism between certain knot groups.
\end{abstract}

AMS Classification 57M25; 57M05

Keywords Twisted Alexander polynomial, finitely presentable group, surjective homomorphism, Reidemeister torsion

\section{Introduction}

Suppose that $G$ is a finitely presentable group with a surjective homomorphism to the free abelian group of rank $l$, eg, abelianization. Let $\rho: G \rightarrow G L(n ; R)$ be a linear representation. The twisted Alexander polynomial of $G$ associated to $\rho$ was introduced in [10] and is defined to be a rational expression of $l$ indeterminates.

Let $\varphi: G \rightarrow G^{\prime}$ be a surjective homomorphism. Each representation $\rho^{\prime}: G^{\prime} \rightarrow$ $G L(n ; R)$ naturally induces a representation of $G$, namely, $\rho=\rho^{\prime} \circ \varphi$. In this paper we prove the following:

Main theorem The twisted Alexander polynomial of $G$ associated to $\rho$ is divisible by the twisted Alexander polynomial of $G^{\prime}$ associated to $\rho^{\prime}$.

The corresponding fact about the Alexander polynomial is known [1].

We present two separate proofs of the main theorem. First we give a purely algebraic proof in $\$ 3$. If $G$ is a knot group, the twisted Alexander polynomial of $G$ may be regarded as the Reidemeister torsion. In we provide another 
proof of the main theorem in case when $G$ and $G^{\prime}$ are knot groups, from the view point of the Reidemeister torsion.

In the last section, we show non-existence of surjective homomorphism between certain knot groups, as an application of the main theorem.

\section{Twisted Alexander polynomial}

In this section, we recall briefly the definition of the twisted Alexander polynomial.

Let $G$ be a finitely presentable group. Choose and fix a presentation as follows:

$$
G=\left\langle x_{1}, \ldots, x_{u} \mid r_{1}, \ldots, r_{v}\right\rangle .
$$

We denote by $\alpha: G \rightarrow \mathbb{Z}^{l}$ a surjective homomorphism to the free abelian group with generators $t_{1}, \ldots, t_{l}$ and $\rho: G \rightarrow G L(n ; R)$ a linear representation, where $R$ is a unique factorization domain. These maps naturally induce ring homomorphisms $\tilde{\rho}$ and $\tilde{\alpha}$ from $\mathbb{Z}[G]$ to $M(n ; R)$ and $\mathbb{Z}\left[t_{1}^{ \pm 1}, \ldots, t_{l}^{ \pm 1}\right]$ respectively, where $M(n ; R)$ denotes the matrix algebra of degree $n$ over $R$. Then $\tilde{\rho} \otimes \tilde{\alpha}$ defines a ring homomorphism

$$
\mathbb{Z}[G] \rightarrow M\left(n ; R\left[t_{1}^{ \pm 1}, \ldots, t_{l}^{ \pm 1}\right]\right) .
$$

Let $F_{u}$ be the free group on generators $x_{1}, \ldots, x_{u}$ and

$$
\Phi: \mathbb{Z}\left[F_{u}\right] \rightarrow M\left(n ; R\left[t_{1}^{ \pm 1}, \ldots, t_{l}^{ \pm 1}\right]\right)
$$

the composite of the surjection $\mathbb{Z}\left[F_{u}\right] \rightarrow \mathbb{Z}[G]$ induced by the fixed presentation and the map $\tilde{\rho} \otimes \tilde{\alpha}: \mathbb{Z}[G] \rightarrow M\left(n ; R\left[t_{1}^{ \pm 1}, \ldots, t_{l}^{ \pm 1}\right]\right)$.

We define the $v \times u$ matrix $M$ whose $(i, j)$ component is the $n \times n$ matrix

$$
\Phi\left(\frac{\partial r_{i}}{\partial x_{j}}\right) \in M\left(n ; R\left[t_{1}^{ \pm 1}, \ldots, t_{l}^{ \pm 1}\right]\right),
$$

where $\partial / \partial x$ denotes the Fox derivation. This matrix $M$ is called the Alexander matrix of the presentation of $G$ associated to the representation $\rho$.

It is easy to see that there is an integer $1 \leq j \leq u$ such that $\operatorname{det} \Phi\left(x_{j}-1\right) \neq 0$. For such $j$, let us denote by $M_{j}$ the $v \times(u-1)$ matrix obtained from $M$ by removing the $j$-th column. We regard $M_{j}$ as an $n v \times n(u-1)$ matrix with coefficients in $R\left[t_{1}^{ \pm 1}, \ldots, t_{l}^{ \pm 1}\right]$. Moreover, for an $n(u-1)$-tuple of indices

$$
I=\left(i_{1}, i_{2}, \ldots, i_{n(u-1)}\right), \quad\left(1 \leq i_{1}<i_{2}<\cdots<i_{n(u-1)} \leq n v\right)
$$


we denote by $M_{j}^{I}$ the $n(u-1) \times n(u-1)$ square matrix consisting of the $i_{k}$-th row of the matrix $M_{j}$, where $k=1,2, \ldots, n(u-1)$.

Then the twisted Alexander polynomial (see [10]) of a finitely presented group $G$ for a representation $\rho: G \rightarrow G L(n ; R)$ is defined to be a rational expression

$$
\Delta_{G, \rho}\left(t_{1}, \ldots, t_{l}\right)=\frac{\operatorname{gcd}_{I}\left(\operatorname{det} M_{j}^{I}\right)}{\operatorname{det} \Phi\left(x_{j}-1\right)}
$$

and moreover is well-defined up to a factor $\epsilon t_{1}{ }^{\varepsilon_{1}} \cdots t_{l} \varepsilon_{l}$, where $\epsilon \in R^{\times}, \varepsilon_{i} \in \mathbb{Z}$. See [10], 7], 2] and [3] for more precise definition and applications.

\section{Main theorem and the algebraic proof}

In this section, we prove the following main theorem of this paper.

Theorem 3.1 Let $G$ and $G^{\prime}$ be finitely presentable groups and $\alpha, \alpha^{\prime}$ surjective homomorphisms from $G, G^{\prime}$ to $\mathbb{Z}^{l}$ respectively. Suppose that there exists a surjective homomorphism $\varphi: G \rightarrow G^{\prime}$ such that $\alpha=\alpha^{\prime} \circ \varphi$. Then $\Delta_{G, \rho}$ is divisible by $\Delta_{G^{\prime}, \rho^{\prime}}$ for any representation $\rho^{\prime}: G^{\prime} \rightarrow G L(n ; R)$, where $\rho=\rho^{\prime} \circ \varphi$. That is to say, the quotient of $\Delta_{G, \rho}$ by $\Delta_{G^{\prime}, \rho^{\prime}}$ is a genuine polynomial.

Proof Choose and fix a presentation

$$
G=\left\langle x_{1}, x_{2}, \ldots, x_{u} \mid r_{1}, r_{2}, \ldots, r_{v}\right\rangle .
$$

Since $\varphi$ is surjective, then $G^{\prime}$ is generated by $\varphi\left(x_{1}\right), \ldots, \varphi\left(x_{u}\right)$. Namely, $G^{\prime}$ can be presented as

$$
G^{\prime}=\left\langle\varphi\left(x_{1}\right), \varphi\left(x_{2}\right), \ldots, \varphi\left(x_{u}\right) \mid s_{1}, s_{2}, \ldots, s_{v^{\prime}}\right\rangle .
$$

For convenience, we also write $x_{i}$ for $\varphi\left(x_{i}\right)$, that is, we consider that $G^{\prime}$ is generated by $x_{1}, \ldots, x_{u}$. By this notation, each relator $r_{i}$ is written as

$$
r_{i}=\prod_{k} u_{k} s_{l_{i_{k}}}^{\varepsilon_{i_{k}}} u_{k}^{-1}, \quad i=1,2, \ldots, v, 1 \leq l_{i_{k}} \leq v^{\prime}, u_{k} \in F_{u}, \varepsilon_{i_{k}}= \pm 1
$$

since $\varphi$ is a homomorphism. By applying the Fox derivation $\frac{\partial}{\partial x_{j}}$ and collecting terms of $\frac{\partial s_{k}}{\partial x_{j}}$, we get

$$
\varphi\left(\frac{\partial r_{i}}{\partial x_{j}}\right)=\sum_{k=1}^{v^{\prime}} A_{i, k} \frac{\partial s_{k}}{\partial x_{j}}
$$


Here $A_{i, k}(1 \leq i \leq v)$ is a sum of some $\varepsilon_{\bullet} \varphi\left(u_{\bullet}\right)$, which does not depend on $j$. Let $M_{G}$ and $M_{G^{\prime}}$ be the Alexander matrices with the $u$-th column removed:

$$
\begin{gathered}
M_{G}=\left(\begin{array}{ccc}
\tilde{\rho} \otimes \tilde{\alpha}\left(\frac{\partial r_{1}}{\partial x_{1}}\right) & \cdots & \tilde{\rho} \otimes \tilde{\alpha}\left(\frac{\partial r_{1}}{\partial x_{u-1}}\right) \\
\vdots & \ddots & \vdots \\
\tilde{\rho} \otimes \tilde{\alpha}\left(\frac{\partial r_{v}}{\partial x_{1}}\right) & \cdots & \tilde{\rho} \otimes \tilde{\alpha}\left(\frac{\partial r_{v}}{\partial x_{u-1}}\right)
\end{array}\right) \\
M_{G^{\prime}}=\left(\begin{array}{ccc}
\tilde{\rho}^{\prime} \otimes \tilde{\alpha}^{\prime}\left(\frac{\partial s_{1}}{\partial x_{1}}\right) & \cdots & \tilde{\rho}^{\prime} \otimes \tilde{\alpha}^{\prime}\left(\frac{\partial s_{1}}{\partial x_{u-1}}\right) \\
\vdots & \ddots & \vdots \\
\tilde{\rho}^{\prime} \otimes \tilde{\alpha}^{\prime}\left(\frac{\partial s_{v^{\prime}}}{\partial x_{1}}\right) & \cdots & \tilde{\rho}^{\prime} \otimes \tilde{\alpha}^{\prime}\left(\frac{\partial s_{v^{\prime}}}{\partial x_{u-1}}\right)
\end{array}\right) .
\end{gathered}
$$

By (1), we have

$$
M_{G}=A M_{G^{\prime}}
$$

where $A=\left(\rho^{\prime}\left(A_{i, k}\right)\right)$ is a $n v \times n v^{\prime}$ matrix. For $I=\left(i_{1}, i_{2}, \ldots, i_{n(u-1)}\right)$, as is easily shown,

$$
\operatorname{det} M_{G}^{I}=\operatorname{det}\left(A^{I} M_{G^{\prime}}\right)=\sum_{K} \pm\left(\operatorname{det} A_{K}^{I}\right)\left(\operatorname{det} M_{G^{\prime}}^{K}\right)
$$

where $K=\left(k_{1}, k_{2}, \ldots, k_{n(u-1)}\right)$ and $A_{K}^{I}$ is the matrix consisting of the $k_{1}, k_{2}$, $\ldots, k_{n(u-1)}$-th columns of $A^{I}$. It follows that if $\operatorname{det} M_{G^{\prime}}^{I}$ has a common divisor $P$ for all $I$, then so does $\operatorname{det} M_{G}^{I}$. Moreover, the denominator of $\Delta_{G, \rho}$ is equal to that of $\Delta_{G^{\prime}, \rho^{\prime}}$. This completes the proof.

The corresponding fact about the Alexander polynomial is well known. Let $G(K)$ be the knot group $\pi_{1}\left(S^{3}-K\right)$ of a knot $K$ in $S^{3}$. For any knots $K, K^{\prime}$, if there exists a surjective homomorphism from $G(K)$ to $G\left(K^{\prime}\right)$, then the Alexander polynomial of $K$ is divisible by that of $K^{\prime}$. Murasugi mentions that if there exists a surjective homomorphism from a knot group $G(K)$ to the trefoil knot group, then the twisted Alexander polynomial of $G(K)$ is divisible by that of the trefoil knot group. The main theorem is a generalization of the above.

We will now make a few remarks about geometric settings in which surjective homomorphisms arise. First we consider the case of degree one maps. Let $X$ and $Y$ be $d$-dimensional compact manifolds. Suppose that $f: X \rightarrow Y$ is a degree one map. It is easy to see that its induced homomorphism $f_{*}: \pi_{1}(X) \rightarrow$ $\pi_{1}(Y)$ is a surjective homomorphism.

In the knot group case, there exist the following situations except for degree 1 maps. First, there exists a surjective homomorphism from any knot group to 
the trivial knot group which is the infinite cyclic group. Secondly, if a knot $K$ is a connected sum of $K_{1}$ and $K_{2}$, then its knot group $G(K)$ is an amalgamated product of $G\left(K_{1}\right)$ and $G\left(K_{2}\right)$. Then there exists a surjection from $G(K)$ to each factor group. Thirdly, if a knot $K$ is a periodic knot of order $n$, then there exists a surjective homomorphism from $G(K)$ to $G\left(K_{*}\right)$ where $K_{*}$ is its quotient knot of $K$.

\section{Another proof from the view point of the Reide- meister torsion}

In this section, we prove our theorem in the knot group case. It is done by using the Mayer-Vietoris argument of the Reidemeister torsion.

Here let us consider a knot $K$ in $S^{3}$ and its exterior $E(K)$. For the knot group $G(K)=\pi_{1} E(K)$, we choose and fix a Wirtinger presentation

$$
G(K)=\left\langle x_{1}, \ldots, x_{u} \mid r_{1}, \ldots, r_{u-1}\right\rangle .
$$

The abelianization homomorphism

$$
\alpha_{K}: G(K) \rightarrow H_{1}(E(K), \mathbb{Z}) \cong \mathbb{Z}=\langle t\rangle
$$

is given by $\alpha_{K}\left(x_{1}\right)=\cdots=\alpha_{K}\left(x_{u}\right)=t$. If we have no confusion, we write simply $\alpha$ for $\alpha_{K}$ as in the previous section. In this section, we take a unimodular representation $\rho: G(K) \rightarrow S L(n ; \mathbb{F})$ over a field $\mathbb{F}$. As in the definition of the twisted Alexander polynomial, we consider the tensor representation

$$
\rho \otimes \alpha: G \rightarrow G L\left(n ; \mathbb{F}\left[t, t^{-1}\right]\right) \subset G L(n ; \mathbb{F}(t)) .
$$

Here $\mathbb{F}(t)$ denotes the rational function field over $\mathbb{F}$. If $\rho \otimes \alpha$ is an acyclic representation over $\mathbb{F}(t)$, that is, all homology groups over $\mathbb{F}(t)$ of $E(K)$ twisted by $\rho \otimes \alpha$ are vanishing, then the Reidemeister torsion of $E(K)$ for $\rho \otimes \alpha$ can be

defined. Furthermore the following equality holds. See [3, 4, for more details of definitions and proofs.

Theorem 4.1 If $\rho \otimes \alpha$ is an acyclic representation, then we have

$$
\tau_{\rho \otimes \alpha}(E(K))=\Delta_{G(K), \rho}(t)
$$

up to a factor $\pm t^{n k}(k \in \mathbb{Z})$ if $n$ is odd, and up to only $t^{n k}$ if $n$ is even.

From this theorem, we prove the main theorem as divisibility of the Reidemeister torsion in the knot group case. Here we take a surjective homomorphism 
$\varphi: G(K) \rightarrow G\left(K^{\prime}\right)$. By changing the orientation of meridians if we need, we may assume that $\alpha_{K^{\prime}} \circ \varphi=\alpha_{K}$. Let $\rho^{\prime}: G\left(K^{\prime}\right) \rightarrow S L(n ; \mathbb{F})$ be a representation. For simplicity, we write the composition $\rho=\rho^{\prime} \circ \varphi$.

Now we consider 2-dimensional CW-complexes $X(K)$ and $X\left(K^{\prime}\right)$ defined by their Wirtinger presentations. It is well-known that these complexes are simple homotopy equivalent to the knot exteriors. Then these Reidemeister torsions of $X(K)$ and $X\left(K^{\prime}\right)$ are equal to the twisted Alexander polynomials respectively. Here we consider twisted homologies of these complexes by using their CWcomplex structure. The coefficient $V$ is a $2 n$-dimensional vector space over a rational function field $\mathbb{F}(t)$. When $V$ is regarded as a $G(K)$-module by using $\rho$, it is denoted by $V_{\rho}$.

The surjective homomorphism $\varphi$ induces a chain map $\varphi_{*}: C_{*}\left(X(K), V_{\rho}\right) \rightarrow$ $C_{*}\left(X\left(K^{\prime}\right), V_{\rho^{\prime}}\right)$. We take a tensor representation $\rho \otimes \alpha_{K}: G(K) \rightarrow G L(n ; \mathbb{F}(t))$. Assume that $\rho \otimes \alpha_{K}$ and $\rho^{\prime} \otimes \alpha_{K^{\prime}}$ are acyclic representations. Then we can prove the following.

Theorem 4.2 The quotient $\tau\left(X(K) ; V_{\rho \otimes \alpha_{K}}\right) / \tau\left(X\left(K^{\prime}\right) ; V_{\rho^{\prime} \otimes \alpha_{K^{\prime}}}\right)$ is a polynomial in $\mathbb{F}\left[t, t^{-1}\right]$.

We show the following proposition first.

Proposition 4.3 The chain map

$$
\varphi_{*}: C_{*}\left(X(K), V_{\rho \otimes \alpha_{K}}\right) \rightarrow C_{*}\left(X\left(K^{\prime}\right), V_{\rho^{\prime} \otimes \alpha_{K^{\prime}}}\right)
$$

is surjective.

Proof It is clear that $\varphi$ induces an isomorphism on the 0-chains, and a surjection on the 1-chains. Then we only need to prove this proposition on the 2-chains.

We take a non-trivial 2-chain $z \in C_{2}\left(X\left(K^{\prime}\right), V_{\rho^{\prime}} \otimes \alpha_{K^{\prime}}\right)$. By the acyclicity of the chain complex $C_{*}\left(X\left(K^{\prime}\right), V_{\rho^{\prime} \otimes \alpha_{K^{\prime}}}\right)$, the boundary map $\partial: C_{2}\left(X\left(K^{\prime}\right), V_{\rho^{\prime}} \otimes \alpha_{K^{\prime}}\right)$ $\rightarrow C_{1}\left(X\left(K^{\prime}\right), V_{\rho^{\prime} \otimes \alpha_{K^{\prime}}}\right)$ is injective. Then the image $\partial z$ is non-trivial in $C_{1}$. On the other hand, by the surjectivity of

$$
\varphi: C_{1}\left(X(K), V_{\rho \otimes \alpha_{K}}\right) \rightarrow C_{1}\left(X\left(K^{\prime}\right), V_{\rho^{\prime} \otimes \alpha_{K^{\prime}}}\right),
$$

there exists a 2-chain $w \in C_{2}\left(X(K), V_{\rho \otimes \alpha_{K}}\right)$ such that $\varphi_{*}(w)=z$. By the commutativity of maps, in $C_{2}$

$$
\varphi_{*}(\partial w)=\partial \varphi_{*}(w)=\partial \partial z=0 .
$$


Then we have $\partial w=0$. By the acyclicity, there exists $\tilde{w} \in C_{*}\left(X(K), V_{\rho \otimes \alpha_{K}}\right)$ such that $\partial \tilde{w}=w$. Again by the commutativity, $\varphi(\tilde{w})=z$. Therefore $\varphi_{*}$ is surjective.

Proof of Theorem 4.2 From the above proposition, we can take the kernel $D_{*}$ of this chain map $\varphi_{*}$ and obtain a short exact sequence

$$
0 \rightarrow D_{*} \rightarrow C_{*}\left(X(K), V_{\rho \otimes \alpha_{K}}\right) \rightarrow C_{*}\left(X\left(K^{\prime}\right), V_{\rho^{\prime} \otimes \alpha_{K^{\prime}}}\right) \rightarrow 0 .
$$

Here we recall the following fact. For a short exact sequence $0 \rightarrow C_{*}^{\prime} \rightarrow C_{*} \rightarrow$ $C_{*}^{\prime \prime} \rightarrow 0$ of finite chain complexes, if two of them are acyclic complexes, then the third one is also acyclic. Furthermore, the torsion satisfies

$$
\tau\left(C_{*}\right)=\tau\left(C_{*}^{\prime}\right) \tau\left(C_{*}^{\prime \prime}\right)
$$

up to some factor.

By applying the property of the product of torsion, we have

$$
\tau\left(X(K) ; V_{\rho \otimes \alpha_{K}}\right)=\tau\left(X\left(K^{\prime}\right) ; V_{\rho^{\prime} \otimes \alpha_{K^{\prime}}}\right) \tau\left(D ; V_{\rho \otimes \alpha_{K}}\right) .
$$

We only need to prove that $\tau\left(D ; V_{\rho \otimes \alpha_{K}}\right)$ is a polynomial. From the definition we see that $D_{0}$ vanishes, since

$$
\varphi_{*}: C_{0}\left(X(K), V_{\rho \otimes \alpha_{K}}\right) \rightarrow C_{0}\left(X\left(K^{\prime}\right), V_{\rho^{\prime} \otimes \alpha_{K^{\prime}}}\right)
$$

is isomorphism. Hence by definition, its torsion is the determinant of $D_{2} \rightarrow D_{1}$. Therefore it is a polynomial.

Remark 4.4 By a similar argument, we can prove that if $\varphi: G(K) \rightarrow G\left(K^{\prime}\right)$ is an injective homomorphism, then $\tau\left(X\left(K^{\prime}\right) ; V_{\rho \otimes \alpha_{K^{\prime}}}\right) / \tau\left(X(K) ; V_{\rho \otimes \alpha_{K}}\right)$ is a polynomial.

\section{$5 \quad$ Examples}

In this section, we show some examples of the twisted Alexander polynomials and an application of Theorem 3.1. We consider the problem: Is there a surjective homomorphism from $G(K)$ to $G\left(K^{\prime}\right)$ for two given knots $K, K^{\prime}$ ? The problem has been investigated by Murasugi when $K^{\prime}$ is the trefoil knot $3_{1}$ (c.f. [8]). Here we study the problem in case when $K^{\prime}$ is the figure eight knot $4_{1}$. The numbering of the knots follows that of Rolfsen's book 9].

If the classical Alexander polynomial of $K$ can not be divided by that of $K^{\prime}$, we know that there are no surjective homomorphisms from $G(K)$ to $G\left(K^{\prime}\right)$. 
In the knot table in 9], up to 9 crossings, the classical Alexander polynomial of each knot is not divisible by that of $G\left(4_{1}\right)$ except for $8_{18}, 8_{21}, 9_{12}, 9_{24}, 9_{37}$, $9_{39}$ and $9_{40}$. That is to say, except for $8_{18}, 8_{21}, 9_{12}, 9_{24}, 9_{37}, 9_{39}$ and $9_{40}$, there exists no surjective homomorphisms from such a knot group to $G\left(4_{1}\right)$.

Next, we consider a representation $\rho: G(K) \rightarrow S L(2 ; \mathbb{Z} / p \mathbb{Z})$ and the twisted Alexander polynomial associated to $\rho$. Theorem 3.1 says that if the numerator of $\Delta_{G(K), \rho}$ for all representations $\rho: G(K) \rightarrow S L(2 ; \mathbb{Z} / p \mathbb{Z})$ for some fixed prime $p$ cannot be divided by the numerator of $\Delta_{G\left(K^{\prime}\right), \rho^{\prime}}$ for a certain representation $\rho^{\prime}: G\left(K^{\prime}\right) \rightarrow S L(2 ; \mathbb{Z} / p \mathbb{Z})$, then there exists no surjective homomorphisms from $G(K)$ to $G\left(K^{\prime}\right)$.

Let us compute the twisted Alexander polynomials $\Delta_{G\left(4_{1}\right), \rho^{\prime}}$ for a certain representation $\rho^{\prime}: G\left(4_{1}\right) \rightarrow S L(2 ; \mathbb{Z} / 7 \mathbb{Z})$. The knot group $G\left(4_{1}\right)$ admits a presentation

$$
G\left(4_{1}\right)=\left\langle x_{1}, x_{2}, x_{3}, x_{4} \mid x_{4} x_{2} x_{4}^{-1} x_{1}^{-1}, x_{1} x_{2} x_{1}^{-1} x_{3}^{-1}, x_{2} x_{4} x_{2}^{-1} x_{3}^{-1}\right\rangle .
$$

We can check easily that the following is a representation of $G\left(4_{1}\right)$ :

$$
\begin{aligned}
& \rho^{\prime}\left(x_{1}\right)=\left(\begin{array}{ll}
1 & 1 \\
0 & 1
\end{array}\right), \rho^{\prime}\left(x_{2}\right)=\left(\begin{array}{ll}
1 & 0 \\
3 & 1
\end{array}\right), \\
& \rho^{\prime}\left(x_{3}\right)=\left(\begin{array}{ll}
4 & 4 \\
3 & 5
\end{array}\right), \rho^{\prime}\left(x_{4}\right)=\left(\begin{array}{ll}
2 & 4 \\
5 & 0
\end{array}\right) .
\end{aligned}
$$

Then we obtain the Alexander matrix:

$$
M=\left(\begin{array}{cccccccc}
6 & 0 & 2 t & 4 t & 0 & 0 & 6 t+1 & 6 t \\
0 & 6 & 5 t & 0 & 0 & 0 & 0 & 6 t+1 \\
3 t+1 & 3 t & t & t & 6 & 0 & 0 & 0 \\
4 t & 2 t+1 & 0 & t & 0 & 6 & 0 & 0 \\
0 & 0 & 3 t+1 & 3 t & 6 & 0 & t & 0 \\
0 & 0 & 4 t & 2 t+1 & 0 & 6 & 3 t & t
\end{array}\right)
$$

The numerator $P$ of the twisted Alexander polynomial $\Delta_{G\left(4_{1}\right), \rho^{\prime}}$ is the determinant of $M_{4}$ obtained from $M$ by removing the last two columns. Then we get

$$
P=t^{4}+t^{3}+3 t^{2}+t+1 \text {. }
$$

Moreover, we calculate the numerator of the twisted Alexander polynomials of $G\left(8_{21}\right)$ for all representations $G\left(8_{21}\right) \rightarrow S L(2 ; \mathbb{Z} / 7 \mathbb{Z})$ and get 24 polynomials. These calculations are made by author's computer program and the same results are obtained by Kodama Knot program [6]. None of them can be divided by $P$, so we conclude that there exists no surjective homomorphisms from $G\left(8_{21}\right)$ to $G\left(4_{1}\right)$. By similar arguments using $S L(2 ; \mathbb{Z} / p \mathbb{Z})$-representations for 
$p=5,7$, we get the conclusion that there exists no surjective homomorphisms from $G\left(9_{12}\right), G\left(9_{24}\right), G\left(9_{39}\right)$ to $G\left(4_{1}\right)$. On the other hand, $8_{18}$ is a periodic knot of order 2 with quotient knot $4_{1}$. Furthermore, $G\left(9_{37}\right)$ has a presentation

$$
\begin{aligned}
& G\left(9_{37}\right)= \\
& \left\langle\begin{array}{c|c}
y_{1}, y_{2}, y_{3}, y_{4}, y_{5}, & y_{8} y_{1} y_{8}^{-1} y_{2}^{-1}, y_{7} y_{2} y_{7}^{-1} y_{3}^{-1}, y_{9} y_{4} y_{9}^{-1} y_{3}^{-1}, y_{3} y_{4} y_{3}^{-1} y_{5}^{-1}, \\
y_{6}, y_{7}, y_{8}, y_{9} & y_{1} y_{6} y_{1}^{-1} y_{5}^{-1}, y_{5} y_{6} y_{5}^{-1} y_{7}^{-1}, y_{2} y_{7} y_{2}^{-1} y_{8}^{-1}, y_{4} y_{9} y_{4}^{-1} y_{8}^{-1}
\end{array}\right\rangle
\end{aligned}
$$

and the following mapping $\varphi: G\left(9_{37}\right) \rightarrow G\left(4_{1}\right)$ is a surjective homomorphism:

$$
\begin{gathered}
\varphi\left(y_{1}\right)=x_{2}, \varphi\left(y_{2}\right)=x_{3}, \varphi\left(y_{3}\right)=x_{1} x_{4} x_{1}^{-1}, \varphi\left(y_{4}\right)=x_{3}, \varphi\left(y_{5}\right)=x_{1}, \\
\varphi\left(y_{6}\right)=x_{1}^{-1} x_{4} x_{1}, \varphi\left(y_{7}\right)=x_{4}, \varphi\left(y_{8}\right)=x_{1}, \varphi\left(y_{9}\right)=x_{4} .
\end{gathered}
$$

Similarly, we can give an explicit surjective homomorphism from the knot group $G\left(9_{40}\right)$ to $G\left(4_{1}\right)$. Thus we have surjective homomorphisms from knot groups $G\left(8_{18}\right), G\left(9_{37}\right), G\left(9_{40}\right)$ to $G\left(4_{1}\right)$. Hence we can determine whether or not there exists a surjective homomorphism from the group of each knot with up to 9 crossings to $G\left(4_{1}\right)$.

In [5], we see a complete list of whether there exists a surjective homomorphism between knot groups for 10 crossings and less.

Acknowledgements The first author is supported in part by Grand-in-Aid for Scientific Research (No. 14740037), The Ministry of Education, Culture, Sports, Science and Technology, Japan. The second author is supported by the 21 century COE program at Graduate School of Mathematical Sciences, the University of Tokyo. The authors would like to express their thanks to Prof. Sadayoshi Kojima and Prof. Dieter Kotschick for their useful comments.

\section{References}

[1] RH Crowell, RH Fox, Introduction to knot theory, Graduate texts 57, Springer-Verlag, New York (1977) MathReview

[2] H Goda, T Kitano, T Morifuji, Reidemeister torsion, twisted Alexander polynomial and fibered knots, Comment. Math. Helv. 80 (2005) 51-61 MathReview

[3] P Kirk, C Livingston, Twisted Alexander invariants, Reidemeister torsion, and Casson-Gordon invariants, Topology 38 (1999) 635-661 MathReview

[4] T Kitano, Twisted Alexander polynomial and Reidemeister torsion, Pacific J. Math. 174 (1996) 431-442 MathReview

[5] T Kitano, M Suzuki, A partial order in the knot table, to appear in Experimental Math. 
[6] K Kodama, http://www.math.kobe-u.ac.jp/HOME/kodama/knot.html

[7] X S Lin, Representations of knot groups and twisted Alexander polynomials, Acta Math. Sin. (Engl. Ser.) 17 (2001) 361-380 MathReview

[8] K Murasugi, Lecture in Osaka City University, (2003)

[9] D Rolfsen, Knots and links, Publish or Perish Inc. Berkeley, Calif. (1976) MathReview

[10] M Wada, Twisted Alexander polynomial for finitely presentable groups, Topology 33 (1994) 241-256 MathReview

Department of Mathematical and Computing Sciences, Tokyo Institute of Technology 2-12-1-W8-43 Oh-okayama, Meguro-ku, Tokyo, 152-8552 Japan

Graduate School of Mathematical Sciences, The University of Tokyo

3-8-1 Komaba, Meguro-ku, Tokyo, 153-8914 Japan

and

Department of Information and Computer Sciences, Nara Women's University

Kita-Uoya Nishimachi, Nara, 630-8506 Japan

Email: kitano@is.titech.ac.jp, macky@ms.u-tokyo.ac.jp and

wada@ics.nara-wu.ac.jp

Received: 6 July 2005 